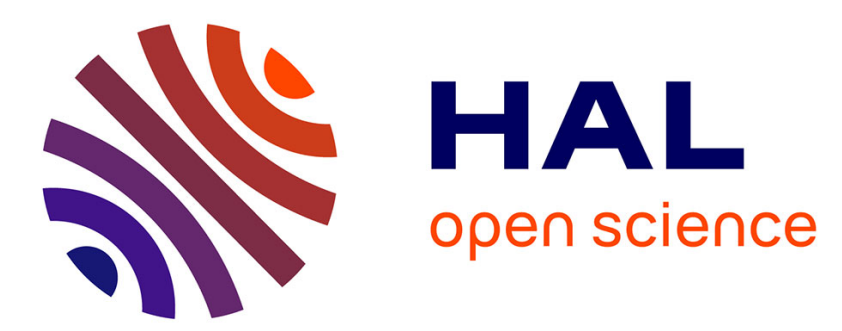

\title{
A SHORT NOTE ON A PAIR OF MEROMORPHIC FUNCTIONS IN A p-ADIC FIELD, SHARING A FEW SMALL ONES
}

Alain Escassut, C. Yang

\section{- To cite this version:}

Alain Escassut, C. Yang. A SHORT NOTE ON A PAIR OF MEROMORPHIC FUNCTIONS IN A p-ADIC FIELD, SHARING A FEW SMALL ONES. 2019. hal-02377422

\section{HAL Id: hal-02377422 \\ https://hal.uca.fr/hal-02377422}

Preprint submitted on 23 Nov 2019

HAL is a multi-disciplinary open access archive for the deposit and dissemination of scientific research documents, whether they are published or not. The documents may come from teaching and research institutions in France or abroad, or from public or private research centers.
L'archive ouverte pluridisciplinaire HAL, est destinée au dépôt et à la diffusion de documents scientifiques de niveau recherche, publiés ou non, émanant des établissements d'enseignement et de recherche français ou étrangers, des laboratoires publics ou privés. 


\title{
A SHORT NOTE ON A PAIR OF MEROMORPHIC FUNCTIONS IN A $p$-ADIC FIELD, SHARING A FEW SMALL ONES
}

\author{
ALAIN ESCASSUT AND C.C. YANG
}

\begin{abstract}
Let $f, g$, be two meromorphic functions on a complete ultrametric algebraically closed field $\mathbb{K}$ of characteristic 0 , or two meromorphic functions in an open disk of $\mathbb{K}$, that are not quotients of bounded analytic functions by polynomials. If $f$ and $g$ share I.M. 9 small meromorphic functions then $f=g$.

If $f$ and $g$ have finitely many poles and share I.M. 4 small meromorphic functions, then $f=g$.
\end{abstract}

\section{Main results}

Let $\mathbb{I K}$ be a complete ultrametric algebraically closed field of characteristic 0 . Let us fix $a \in \mathbb{K}$ and let $R \in] 0,+\infty\left[\right.$. We denote by $d\left(a, R^{-}\right)$the disk $\{x \in \mathbb{K}|| x-a \mid<R\}$.

We denote by $\mathcal{A}(\mathbb{K})$ the $\mathbb{K}$-algebra of entire functions in $\mathbb{K}$ and by $\mathcal{M}(\mathbb{K})$ the field of meromorphic functions which is its field of fractions. We denote by $\mathcal{A}\left(d\left(a, R^{-}\right)\right)$the $\mathbb{K}$-algebra of analytic functions in $d\left(a, R^{-}\right)$i.e. the set of power series converging in the disk $d\left(a, R^{-}\right)$. and by $\mathcal{M}\left(d\left(a, R^{-}\right)\right)$the field of meromorphic functions in $d\left(a, R^{-}\right)$i.e. the field of fractions of $\mathcal{A}\left(d\left(a, R^{-}\right)\right)$. Moreover, we denote by $\mathcal{A}_{b}\left(d\left(a, R^{-}\right)\right)$the $\mathbb{K}$-algebra of functions $f \in \mathcal{A}\left(d\left(a, R^{-}\right)\right)$ that are bounded in $d\left(a, R^{-}\right)$, by $\mathcal{M}_{b}\left(\left(a, R^{-}\right)\right)$its field of fractions and we put $\left.\mathcal{M}_{u}\left(d\left(a, R^{-}\right)\right)=\mathcal{M}\left(\left(a, R^{-}\right)\right) \backslash \mathcal{A}_{b}\left(a, R^{-}\right)\right)$

We define $N(r, f)^{\prime}([1]$, chapter 40 or [3], chapter 2$)$ in the same way as for complex meromorphic functions [2]. Let $f$ be a meromorphic function in all $\mathbb{K}$ having no zero and no pole at 0 . Let $\left(a_{n}\right)_{n \in \mathbb{N}}$ be the sequence of poles of $f$, of respective order $s_{n}$, with $\left|a_{n}\right| \leq\left|a_{n+1}\right|$ and, given $r>0$, (resp. $\left.r \in\right] 0, R[)$, let $q(r)$ be such that $\left|a_{q(r)}\right| \leq r,\left|a_{q(r)+1}\right|>r$. We then denote by $N(r, f)$ the counting function of the zeros of $f$, counting multiplicity, as usual: for all $r>0$, we put $N(r, f)=\sum_{j=0}^{q(r)} s_{j}\left(\log \left|\left(a_{J}\right)\right|-\log (r)\right)$. Moreover, we denote by $\bar{N}(r, f)$ the counting function of the poles of $f$, ignoring multiplicity as $\sum_{j=0}^{q(r)}\left(\log \left|\left(a_{J}\right)\right|-\log (r)\right)$. Next, we define the counting function of zeros of $f$ as $Z(r, f)=N\left(r, \frac{1}{f}\right)$ and we put $\bar{Z}(r, f)=\bar{N}\left(r, \frac{1}{f}\right)$. Finally we put $T(r, f)=\max (Z(r, f), N(r, f))$. Then

\footnotetext{
${ }^{0}$ Keywords: p-adic meromorphic functions, small functions, sharing small functions 1991 Mathematics Subject Classification. Primary 12J25; Secondary 30D35, 30 G06.
} 
$T(r, f)$ is strictly increasing and has most of properties of the characteristic function of a complex function, concerning operations (see [1], chapter 40 and [3], chapter 2.)

A function $w \in \mathcal{M}(\mathbb{K})$ (resp. $w \in \mathcal{M}\left(d\left(a, R^{-}\right)\right)$) is called a small function with respect to $f$ if $\lim _{r \rightarrow+\infty} \frac{T(r, w)}{T(r, f)}=0$ (resp. $\lim _{r \rightarrow R^{-}} \frac{T(r, w)}{T(r, f)}=0$ ) and we denote by $\mathcal{M}_{f}(\mathbb{K})$ (resp.

$\left.\mathcal{M}_{f}\left(d\left(a, R^{-}\right)\right)\right)$the set of small functions with espect to $f$.

Two functions $f, g \in \mathcal{M}(\mathbb{K})$ (resp. $f, g \in \mathcal{M}_{u}\left(d\left(a, R^{-}\right)\right)$) are said to share I.M. a small function $w \in \mathcal{M}_{f}(\mathbb{K}) \cap \mathcal{M}_{g}(\mathbb{K})\left(\right.$ resp. $\left.w \in \mathcal{M}_{f}\left(d\left(a, R^{-}\right)\right) \cap \mathcal{M}_{g}\left(d\left(a, R^{-}\right)\right)\right)$ if $f(z)=w(z)$ holds if and only if $g(z)=w(z)$.

Here we aim at studying the problem of two meromorphic functions sharing I.M. a few small meromorphic functions in order to show that these two functions are equal. Indeed thanks to Yamanoi's Nevanlinna Second Main Theorem, a similar result is known in complex analysis when two meromorphic functions share 5 small meromorphic functions. But in p-adic analysis, no Theorem similar to Yamanoi's Theorem is known. Thus, we have studied and used tools or arguments other than Yamanoi's Theorem to derive some similar results as follows.

Theorem 1: Let $f, g \in \mathcal{M}(\mathbb{K})$ be transcendental (resp. $f, g \in \mathcal{M}_{u}\left(d\left(a, R^{-}\right)\right)$) and share I.M. $q$ small functions $w_{j} \in \mathcal{M}_{f}(\mathbb{K}) \cap \mathcal{M}_{g}(\mathbb{K})(j=1, \ldots, q)$ $\left(\right.$ resp. $\left.w_{j} \in \mathcal{M}_{f}\left(d\left(a, R^{-}\right)\right) \cap \mathcal{M}_{g}\left(d\left(a, R^{-}\right)\right)(j=1, \ldots, q)\right)$. Then

$$
\sum_{j=1}^{q} \bar{Z}\left(r, f-w_{j}\right) \leq \bar{Z}(r, f-g)+o(T(r, f))+o(T(r, g)) .
$$

Theorem 2: Let $f, g \in \mathcal{M}(\mathbb{K})$ be transcendental (resp. $f, g \in \mathcal{M}_{u}\left(d\left(a, R^{-}\right)\right)$) and share I.M. 9 distinct small functions (other than the constant $\infty) w_{j} \in$ $\mathcal{M}_{f}(\mathbb{K}) \cap \mathcal{M}_{g}(\mathbb{K})(j=1, \ldots, 9)$ (resp. $w_{j} \in \mathcal{M}_{f}\left(d\left(a, R^{-}\right)\right) \cap \mathcal{M}_{g}\left(d\left(a, R^{-}\right)\right)(j=$ $1, \ldots, 9)$ ). Then $f=g$.

Theorem 3: Let $f, g \in \mathcal{M}(\mathbb{K})$ be transcendental (resp. $f, g \in \mathcal{M}_{u}\left(d\left(a, R^{-}\right)\right.$)) have finitely many poles and share I.M. 4 distinct small functions (other than the constant $\infty) w_{j} \in \mathcal{M}_{f}(\mathbb{K}) \cap \mathcal{M}_{g}(\mathbb{K})(j=1, \ldots, 4)$ (resp. $w_{j} \in \mathcal{M}_{f}\left(d\left(a, R^{-}\right)\right) \cap$ $\left.\mathcal{M}_{g}\left(d\left(a, R^{-}\right)\right)(j=1, \ldots, 4)\right)$. Then $f=g$.

Corollary: Let $f, g \in \mathcal{A}(\mathbb{K})$ be transcendental (resp. $f, g \in \mathcal{A}_{u}\left(d\left(a, R^{-}\right)\right)$) share I.M. 4 distinct small functions (other than the constant $\infty$ ) $w_{j} \in \mathcal{M}_{f}(\mathbb{K}) \cap$ $\mathcal{M}_{g}(\mathbb{K})(j=1, \ldots, 4)$

$\left(\right.$ resp. $\left.w_{j} \in \mathcal{M}_{f}\left(d\left(a, R^{-}\right)\right) \cap \mathcal{M}_{g}\left(d\left(a, R^{-}\right)\right)(j=1, \ldots, 4)\right)$. Then $f=g$.

Remark: The results known in complex analysis suggest that the number 9 obtained in Theorem 2 might be improved, concerning $p$-adic meromorphic functions. On the contrary, concerning analytic functions or meromorphic functions with finitely many poles, the number 4 obtained in Theorem 3 seems the best possible. 


\section{The proofs}

In order to prove the theorems, we need to recall the following three lemmas. Lemma 1 comes from Theorem 40.8 in [1].

Lemma 1: Let $f, g \in \mathcal{M}(\mathbb{K})$ (resp. let $f, g \in \mathcal{M}\left(d\left(a, R^{-}\right)\right)$). Then $T(r, f+$ $g) \leq T(r, f)+T(r, g)+O(1)$.Moreover, if $f$ and $g$ have finitely many poles then $T(r, f+g) \leq \max (T(r, f), T(r, g))+O(\log (r))$.

The following Lemma 2 is Lemma 40.10 in [1] (see also Proposition 2.5 in [3]):

Lemma 2: Let $f \in \mathcal{M}(\mathbb{K})$. Then $T(r, f) \leq O(\log (r))$ in $] 0,+\infty[$ if and only if $f$ belongs to $\mathbb{K}(x)$. Let $f \in \mathcal{M}\left(d\left(a, R^{-}\right)\right)$. Either $f \in \mathcal{M}_{b}\left(d\left(a, R^{-}\right)\right)$and then $T(r, f)$ is bounded in $] 0, R\left[\right.$ or $f \in \mathcal{M}_{u}\left(d\left(a, R^{-}\right)\right)$and then $\lim _{r \rightarrow R^{-}} T(r, f)=+\infty$.

The following Lemma 3 comes from Theorem 43.10 in [1] (see also Theorem 2.21 in [3]).

Lemma 3: Let $f \in \mathcal{M}(\mathbb{K})$ (resp. let $f \in \mathcal{M}_{u}\left(d\left(a, R^{-}\right)\right)$) and let $w_{1}, w_{2}, w_{3} \in$ $\mathcal{M}_{f}(\mathbb{K})$ (resp. let $w_{1}, w_{2}, w_{3} \in \mathcal{M}_{f}\left(d\left(a, R^{-}\right)\right)$). Then

$$
T(r, f) \leq \bar{Z}\left(r, f-w_{1}\right)+\bar{Z}\left(r, f-w_{2}\right)+\bar{Z}\left(r, f-w_{3}\right)+o(T(r, f)) .
$$

The following Lemma 4 comes from Corollary 43.12 in [1].

Lemma 4: Let $f \in \mathcal{M}(\mathbb{K})$ (resp. let $f \in \mathcal{M}_{u}\left(d\left(a, R^{-}\right)\right)$) have finitely many poles and let $w_{1}, w_{2} \in \mathcal{M}_{f}(\mathbb{K})$ (resp. let $w_{1}, w_{2} \in \mathcal{M}_{f}\left(d\left(a, R^{-}\right)\right)$). Then

$$
T(r, f) \leq \bar{Z}\left(r, f-w_{1}\right)+\bar{Z}\left(r, f-w_{2}\right)+o(T(r, f)) .
$$

Proof of Theorem 1: Suppose that $f$ and $g$ belong to $\mathcal{M}(\mathbb{K})$, are not identical and share I.M. $q$ small functions $w_{j} \in \mathcal{M}_{f}(\mathbb{K}) \cap \mathcal{M}_{g}(\mathbb{K})(j=1, \ldots, q)$ (resp. $\left.w_{j} \in \mathcal{M}_{f}\left(d\left(a, R^{-}\right)\right) \cap \mathcal{M}_{g}\left(d\left(a, R^{-}\right)\right)(j=1, \ldots, q)\right)$.

Lat $b$ be a zero of $f-w_{i}$ for a certain index $i$. Then it is also a zero of $g-w_{i}$.

Suppose that $b$ is counted several times in the sum $\sum_{j=1}^{q} \bar{Z}\left(r, f-w_{j}\right)$, which means that it is a zero of another function $f-w_{h}$ for a certain index $h \neq i$. Then we have $w_{i}(b)=w_{h}(b)$ and hence $b$ is a zero of the function $w_{i}-w_{h}$ which belongs to $\mathcal{M}_{f}(\mathbb{K})$. Now, put $\widetilde{Z}\left(r, f-w_{1}\right)=\bar{Z}\left(r, f-w_{1}\right)$ and for each $j>1$, let $\widetilde{Z}\left(r, f-w_{j}\right)$ be the counting function of zeros of $f-w_{j}$ in the disk $d\left(a, r^{-}\right)$ ignoring multiplicity and avoiding the zeros already counted as zeros of $f-w_{h}$ for some $h<j$. Consider now the sum $\sum_{j=1}^{q} \widetilde{Z}\left(r, f-w_{j}\right)$. Since the functions $w_{I}-w_{j}$ belong to $\mathcal{M}_{f}(\mathbb{K})$, clearly, we have

$$
\sum_{j=1}^{q} \bar{Z}\left(r, f-w_{j}\right)=\sum_{j=1}^{q} \widetilde{Z}\left(r, f-w_{j}\right)=o(T(r, f)
$$


It's clear, from the assumption, that $f(x)-w_{j}(x)=0$ impliesg $(x)-w_{j}(x)=0$ and hence $f-g=0$. It follows that

$$
\sum_{j=1}^{q} \bar{Z}\left(r, f-w_{j}\right) \leq \bar{Z}(r, f-g) .
$$

Consequently,

$$
\sum_{j=1}^{q} \bar{Z}\left(r, f-w_{j}\right) \leq \bar{Z}(r, f-g)+o(T(r, f))+o(T(r, g)) .
$$

Now, if $f$ and $g$ belong to $\mathcal{M}\left(d\left(a, R^{-}\right)\right)$, the proof is exactly the same.

Proof of Theorems 2 and 3: Consider first the hypothese of Theorem 2. Suppose that $f$ and $g$ are not identical and share 9 small functions $w_{j} \in \mathcal{M}_{f}(\mathbb{K}) \cap$ $\mathcal{M}_{g}(\mathbb{K})(j=1, \ldots, 9)\left(\right.$ resp. $\left.w_{j} \in \mathcal{M}_{f}\left(d\left(a, R^{-}\right)\right) \cap \mathcal{M}_{g}\left(d\left(a, R^{-}\right)\right)(j=1, \ldots, 9)\right)$. Let us now put $M(r)=\max (T(r, f), T(r, g))$. By Lemma 3, we can write

$$
\begin{aligned}
& T(r, f) \leq \bar{Z}\left(r, f-w_{1}\right)+\bar{Z}\left(r, f-w_{2}\right)+\bar{Z}\left(r, f-w_{3}\right)+o(T(r, f)), \\
& T(r, f) \leq \bar{Z}\left(r, f-w_{4}\right)+\bar{Z}\left(r, f-w_{5}\right)+\bar{Z}\left(r, f-w_{6}\right)+o(T(r, f))
\end{aligned}
$$

and

$$
T(r, f) \leq \bar{Z}\left(r, f-w_{7}\right)+\bar{Z}\left(r, f-w_{8}\right)+\bar{Z}\left(r, f-w_{9}\right)+o(T(r, f))
$$

hence by Theorem 1 ,

$$
3 T(r, f) \leq \sum_{j=1}^{9} T\left(r, f-w_{j}\right)+o(T(r, f)) \leq \bar{Z}(r, f-g)+o(T(r, f))+o(T(r, g))
$$

and similarly,

$$
\left.3 T(r, g) \leq \sum_{j=1}^{9} T\left(r, g-w_{j}\right)\right)+o(T(r, g) \leq \bar{Z}(r, f-g)+o(T(r, f))+o(T(r, g))
$$

therefore

$$
3 M(r) \leq T(r, f-g)+o(M(r)) .
$$

But by Lemma 1, $T(r, f-g) \leq T(r, f)+T(r, g)+O(1) \leq 2 M(r)+O(1)$, therefore by (1) we obtain $3 M(r) \leq 2 M(r)+o(M(r))$.

Now, if $f$ and $g$ are transcendental meromorphic functions, this is absurd by Lemma 2 and proves that $f$ and $g$ are identical. And if $f$ and $g$ belong to $\mathcal{M}_{u}\left(d\left(a, R^{-}\right)\right)$, then by Lemma 4 this is absurd because $r$ is then bounded. That finishes the proof of Theorem 2.

Consider now the hypothese of Theorem 3 and suppose that $f$ and $g$ are meromorphic transcendental functions with finitely many poles, (resp. belong to $\mathcal{M}_{u}\left(d\left(a, R^{-}\right)\right)$with finitely many poles), and share 4 small functions $w_{j} \in$ $\mathcal{M}_{f}(\mathbb{K}) \cap \mathcal{M}_{g}(\mathbb{K})(j=1, \ldots, 4)\left(\right.$ resp. $w_{j} \in \mathcal{M}_{f}\left(d\left(a, R^{-}\right)\right) \cap \mathcal{M}_{g}\left(d\left(a, R^{-}\right)\right)(j=$ $1, \ldots, 4))$. By Lemma 4 , we can write

$$
T(r, f) \leq \bar{Z}\left(r, f-w_{1}\right)+\bar{Z}\left(r, f-w_{2}\right)+o(T(r, f)),
$$


and

$$
T(r, f) \leq \bar{Z}\left(r, f-w_{3}\right)+\bar{Z}\left(r, f-w_{4}\right)+o(T(r, f))
$$

hence by Theorem 1 ,

$$
2 T(r, f) \leq \sum_{j=1}^{4} T\left(r, f-w_{j}\right)+o(T(r, f)) \leq \bar{Z}(r, f-g)+o(T(r, f))+o(T(r, g))
$$

and similarly,

$$
2 T(r, g) \leq \sum_{j=1}^{4} T\left(r, g-w_{j}\right)+o(T(r, g) \leq \bar{Z}(r, f-g)+o(T(r, f))+o(T(r, g))
$$

therefore

$$
2 M(r) \leq T(r, f-g)+o(M(r)) .
$$

But since $f$ and $g$ have finitely many poles, by Lemma 1 we have $T(r, f-g) \leq$ $M(r)+O(1)$. Therefore by (2) we obtain $2 M(r) \leq M(r)+o(M(r))$, which is absurd in the same way as for Theorem 2 and ends the proof of Theorem 3.

\section{REFERENCES}

1. Escassut, A. Value Distribution in p-adic Analysis. World Scientific Publishing Co. Pte. Ltd. Singapore, (2015).

2. Hayman, W.K. Picard values of meromorphic functions and their derivatives, Annals of Mathematics. Vol. 70, no. 1, pp. 9-42 (1959).

3. Hu P.C. and Yang, C.C. Meromorphic Functions over non-Archimedean Fields, Kluwer Academic Publishers (2000).

4. Yamanoi, $\mathbf{K}$ The second main theorem for small functions and related problems, Acta Mathematica 192, p. 225-294 (2004).

${ }^{1}$ Université Clermont Auvergne, UMr CNRS 6620, LMBP, F-63000 ClermontFERRAND, FRANCE.

E-mail address: alain.escassut@uca.fr

2 Université Clermont Auvergne, UMr CNRS 6620, LMBP, F-63000 ClermontFERRAND, FRANCE.

E-mail address: maccyang@163.com 